\title{
Terminal Arbors of Axons Projecting to the Somatosensory Cortex of the Adult Rat. I. The Normal Morphology of Specific Thalamocortical Afferents
}

\author{
Karl F. Jensen ${ }^{1}$ and Herbert P. Killackey ${ }^{2}$ \\ 1.2Department of Psychobiology and 'Department of Anatomy and Neurobiology, University of California, Irvine, California \\ 92717; and 'Division of Neurotoxicology, Health Effects Research Laboratory, U.S. Environmental Protection Agency, \\ Research Triangle Park, North Carolina 27711
}

\begin{abstract}
A characteristic feature of the rat somatosensory neocortex is a discrete topographic representation of the facial whiskers. Afferent fibers projecting to this vibrissae representation were "bulk-labeled" by injecting horseradish peroxidase into the white matter. Terminal arbors with the morphological characteristics of Lorente de No's (1949) "specific" thalamocortical afferents were then reconstructed through serial sections. These terminal arbors, characterized by the discrete organization of their dense plexus in layer IV, have a laminar distribution of boutons that parallels the laminar pattern of terminal degeneration resulting from lesions of the ventral posterior nucleus of the thalamus. The regional distribution of different-sized arbors corresponds to the distribution of vibrissae-related clusters of different sizes. Larger arbors were found in the posteromedial region corresponding to the mystacial vibrissae representation, while smaller arbors were found in the anterolateral region corresponding to the representation of the anterior sinus hairs. Terminal arbors were also reconstructed from sections stained simultaneously to demonstrate the pattern of vibrissae-related clusters. The greatest concentration of boutons on these axons occurred within a single vibrissae-related cluster. Furthermore, when 2 fibers terminated within a single cluster, their terminal arbors appeared to be largely coextensive. The morphology, size, and distribution of these terminal arbors support the hypothesis that the layer IV plexus of a single specific thalamocortical afferent tends to fill a vibrissae-related cluster. Thus, the organization of specific thalamocortical afferents may be responsible for clustered organization within the somatotopic map of the rodent neocortex.
\end{abstract}

The relationship between thalamocortical axons and their cortical targets remains a basic question of neocortical organization. This question has at least 2 components. The first of these is

Received June 30, 1986; revised May 26, 1987; accepted May 26, 1987.

This work was supported by NSF Grant 84-18715 to H.P.K. and NIH Postdoctoral Fellowship NSO6651 to K.F.J.

This manuscript has been reviewed by the Health Effects Research Laboratory, U.S. Environmental Protection Agency, and approved for publication. Mention of trade names or commercial products does not constitute endorsement or recommendation for use.

Correspondence should be addressed to Dr. Karl F. Jensen, Neurotoxicology Division, MD 74-B, Health Effects Research Laboratory, U.S. Environmental Protection Agency, Research Triangle Park, NC 27711.

Copyright (c) 1987 Society for Neuroscience 0270-6474/87/113529-15\$02.00/0 the distribution of thalamic axon terminals with respect to the neocortical layers. The second is the areal distribution of thalamic axons and their relation to cortical maps of the peripheral receptor surfaces. The discrete cytoarchitectonic organization of the rodent somatosensory cortex makes it an ideal site in which to address these questions. A prominent feature of the somatosensory neocortex of a number of rodent species is a discretc topographic representation of portions of the body surface, which is easily visualized with routine anatomical techniques (Woolsey and Van der Loos, 1970; Welker and Woolsey, 1974; Killackey et al., 1976; Killackey and Belford, 1980; Dawson and Killackey, 1987).

Woolsey and Van der Loos (1970) were the first to describe the pattern of discrete cytoarchitectonic units within the mouse somatosensory cortex and to relate their distribution pattern to the peripheral receptor surface. These cytoarchitectonic units, which Woolsey and Van der Loos (1970) termed "barrels," are found in cortical layer IV and are composed of small neurons aggregated into well-defined clusters. Woolsey and Van der Loos suggested that a "barrel" was the cortical correlate of a peripheral receptor unit, a mystacial vibrissa. Physiological studies have since established that the majority of neurons within a "barrel" respond to the stimulation of only a single vibrissa (Welker, 1971, 1976; Simons, 1978; Simons and Woolsey, 1979). Furthermore, the number of neurons in a single "barrel" is proportional to the number of myelinated trigeminal nerve fibers innervating the corresponding vibrissa (Lee and Woolsey, 1975; Welker and Van der Loos, 1986). Thus, the size of a given "barrel" is related to the peripheral innervation density of the corresponding vibrissa.

This discrete organization within cortex is dependent upon an intact periphery during development (Van der Loos and Woolsey, 1973). There is a similar discrete organization, alterable by neonatal peripheral damage, at each synaptic level of the pathway from the sensory periphery to the neocortex (Belford and Killackey, 1979a, b, 1980; Bates et al., 1982; Erzurumlu and Killackey, 1983; Bates and Killackey, 1985). These observations have been interpreted as suggesting that the sensory periphery, acting through afferent pathways, plays a major role in the formation of neocortical representations of sensory surfaces (Killackey, 1980). Consistent with this hypothesis, thalamocortical afferents from the ventroposterior nucleus to layer IV are organized into discrete clusters (Killackey, 1973; Killackey and Leshin, 1975) and the pattern of thalamocortical terminations in layer IV can be altered by neonatal peripheral 


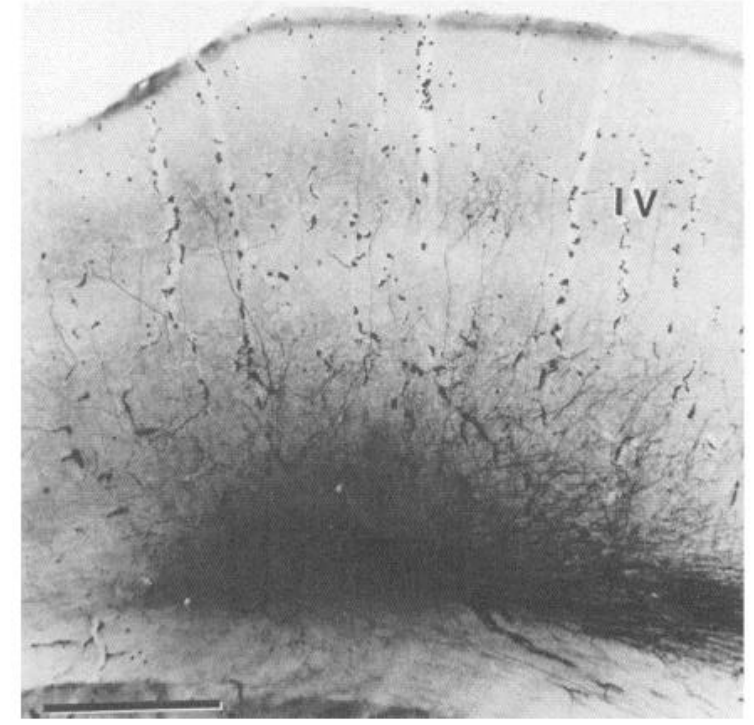

Figure 1. Low-power photomicrograph of a coronal section of the anterolateral region of the somatosensory neocortex illustrating a WGAHRP injection into the junction of the white matter and layer VI. Bulklabeled fibers can be seen traversing the deeper layers and branching in layer IV $(I V)$. Lateral is to the left. Diaminobenzidine. Scale bar, 500 $\mu \mathrm{m}$.

damage (Killackey et al., 1976; Killackey and Belford, 1979). An understanding of the mechanisms that underlie these changes would be greatly aided by a knowledge of the morphology of individual thalamocortical axons.

The morphology of afferents to the somatosensory cortex of the mouse was described by Lorente de No (1922) in his now classic Golgi study. He described afferents that ascend through layers VI and V and branch repeatedly in layer IV to form a dense plexus that extends into layer III. The layer IV plexus of these afferents is organized into clusters, or "glomeruli." The collaterals of a single parent fiber branch extensively in layer IV to form a uniformly dense terminal plexus that appears separated from those in adjacent glomeruli. This description of afferents to cortex was the basis for Lorente de No's (1949) classification of thalamocortical afferents into 2 types. The first type, the "unspecific" or "pluriareal" afferents, give off collaterals in the white matter that innervate multiple cortical fields and have sparse terminals in all cortical layers. The second type, the "specific" thalamocortical afferents, innervate a single cortical field and are characterized by a dense terminal plexus in layer IV.

In the present investigation, we have examined terminal axonal arbors characteristic of the "specific" thalamocortical afferents in the somatosensory cortex of the adult rat. The objective was to determine how the morphology of individual terminal axonal arbors is related to the clustered organization of the somatotopic map of the rodent neocortex. Three approaches were used. First, the laminar organization of boutons of the terminal arbors was compared to the laminar organization of terminal degeneration following lesions of the ventroposterior nucleus. Second, the size of terminal arbors in different regions of the somatosensory cortex was compared to the size of vibrissae-related clusters. Third, arbors were reconstructed in tangential sections stained simultaneously to demonstrate the vibrissae-related clusters.

Abstracts of this work were presented at the Society of Neuroscience Annual Meetings in 1983, 1984, and 1985.

\section{Materials and Methods}

Animals. This study was based on observations in 38 adult female Sprague-Dawley rats. The rats were anesthetized with a combination of ketamine hydrochloride $(50 \mathrm{mg} / \mathrm{kg})$, xylazine $(3 \mathrm{mg} / \mathrm{kg})$, and sodium pentobarbital $(10 \mathrm{mg} / \mathrm{kg})$. WGA-HRP was pressure-injected by means of stereotaxically placed micropipettes (Amaral and Price, 1983). The tip diameter of the micropipettes ranged from 15 to $35 \mu \mathrm{m}$. The concentration of WGA-HRP varied between 5 and $20 \%$ in distilled water and volumes between 0.05 and $0.25 \mu \mathrm{l}$ were injected. A posterior approach was used for injections of WGA-HRP into the internal capsule beneath the somatosensory cortex. The optimal angle of the injection pipette appeared to be approximately $15^{\circ}$ above the horizontal plane, entering the cortex in the occipital region. This approach allowed the tip of the pipette to transect fibers in the white matter and, in some cases, fibers at the junction of the white matter and layer VI (Fig. 1). Survival times ranged from 4 to $24 \mathrm{hr}$. Animals were then intracardially perfused under ether anesthesia according to the method of Rosene and Mesulam (1978). The brain was removed and allowed to sink in $20 \%$ sucrose overnight. Additional animals were perfused in the same manner for demonstration of cytochrome oxidase activity.

Histology. For demonstrations of HRP-filled fibers, the brains were sectioned coronally on a freezing microtome at either 60 or $100 \mu \mathrm{m}$. The WGA-HRP was visualized by the method of Itoh et al. (1979) with diaminobenzidine. To demonstrate simultaneously the terminal arbor morphology and the pattern of vibrissae-related clusters, hemispheres were sectioned in a plane tangential to the cortical surface. Following the HRP reaction, the tangential sections were rinsed 3 times in phosphate buffer and reacted for cytochrome oxidase activity (Wong-Riley, 1979; Land and Simons, 1985). Hemispheres from additional animals were flattened, sectioned tangentially, and reacted for cytochrome oxidase activity.

The laminar distribution of HRP-filled fibers was compared with the laminar distribution of terminal degeneration of thalamocortical afferents, as determined by the Fink-Heimer method, following lesions of the ventral posterior nucleus. Flattened sections of the neocortex stained for succinate dehydrogenase activity were also examined. These materials were from the laboratory collection and the procedures have been previously described (Killackey, 1973; Killackey et al., 1976).

Reconstruction. Individual terminal arbors were drawn using an oilimmersion $40 \times$ objective and an oil-immersion condenser. Branch points and fiber intersections were checked with an oil-immersion $100 \times$ objective. Well-filled terminal arbors of "specific" thalamocortical afferents were characterized by a dense plexus in layer IV and distinctly visible higher-order branches, ending with boutons. Emphasis was placed on arbors whose parent fiber could be traced to layer VI or the white matter. The fibers illustrated were reconstructed over 2-10 sections. Sections were first aligned at lower magnification, and then all visible cut ends were used to align fields at higher magnification. Only unequivocal branches were drawn. This procedure resulted in a presumably conservative image of an axonal arbor that we interpret as representing the significant aspects of its terminal field.

After all fibers were drawn, coverslips were removed and the tissue was counterstained with cresyl violet. The layers were then recorded on the drawings. The drawings were mechanically reduced by $50 \%$ with a pantograph prior to being photographed.

The anteroposterior location of each terminal arbor reconstructed from coronal sections was determined by counting the number of sections from histological landmarks (i.e., crossing of the anterior commissure) and determining their location relative to bregma. The mediolateral location of the terminal arbors was determined by measuring curved (parallel to the pial surface) distance from the longitudinal fissure. This procedure results in slightly larger estimates of the mediolateral extent of the primary somatosensory cortex $(\mathrm{SmI} ; 1.5-8.5 \mathrm{~mm}$ lateral to bregma) than are found in physiologically determined maps of SmI (1.5-7.5 mm lateral to bregma; Chapin and Lin, 1984). Thus, to provide stereotaxic coordinates for a comparison of the location of the terminal arbors to the location of vibrissae-related clusters demonstrated in sections of flattened cortex, small electrolytic lesions were placed at various stereotaxic coordinates in an additional animal, and the cortex flattened, sectioned tangentially, and stained for succinate dehydrogenase activity. The location and size of the layer IV plexus of the terminal arbors were then plotted on a surface view of the somatosensory neocortex for comparison with the location and size of vibrissae-related clusters demonstrated with succinate dehydrogenase or cytochrome oxidase histochemistry. 

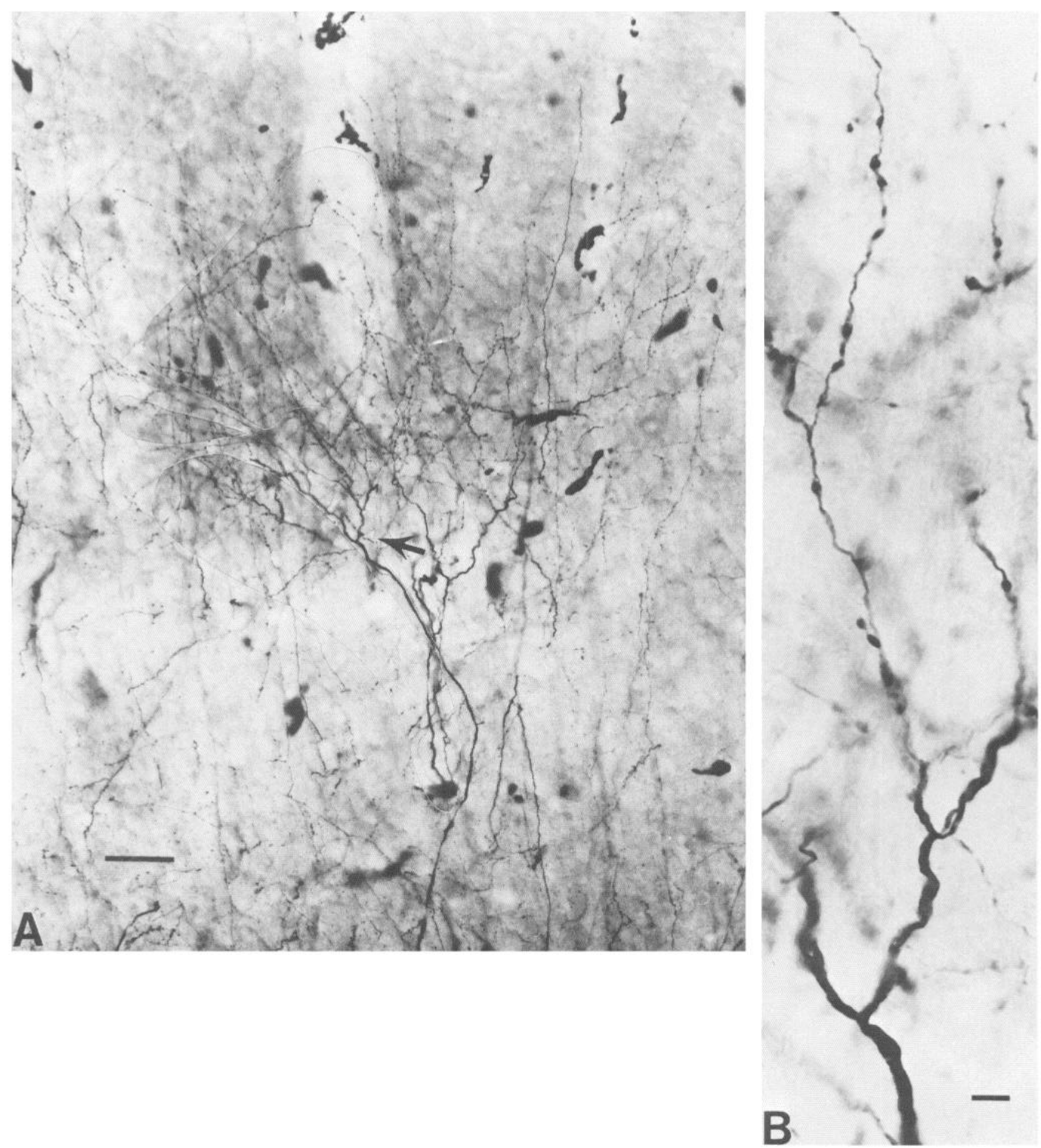

Figure 2. A, Photomicrograph of the layer IV plexus of terminal arbor reconstructed in Figure $4 B, b$. Arrow indicates branch enlarged in $B$ and is located at the junction of layers Va and IV. Top of the figure is the plial surface of the cortex; see Figure $4 B, b$ for laminar boundaries. Diaminobenzidine. Scale bar, $50 \mu \mathrm{m}$. B. Branch with a layer IV plexus of a terminal arbor illustrating the appearance of boutons on the fine-caliber collaterals. Diaminobenzidine. Scale bar, $5 \mu \mathrm{m}$.

Quantitative analysis. The tangential area of layer IV occupied by individual axons was estimated from the mediolateral and anteroposterior extent of the layer IV plexus. The mediolateral extent of the layer IV terminal plexus was measured directly from reconstructions and the maximum anteroposterior extent was estimated from the number of coronal sections the terminal arbor occupied. An estimate of the tangential area of the layer IV plexus was then calculated from the formula for the area of an ellipse, using the mediolateral and anteroposterior measurements as the long and short axes. An analogous estimate was made of the clusters of cytochrome oxidase activity from tangentially sectioned flattened neocortex.

The density of branching in the layer IV terminal plexuses was estimated from drawings by using probe lines spaced $25 \mu \mathrm{m}$ apart and parallel to the pial surface. The number of branches intersecting the probe lines was divided by the length of the probe lines overlaying the terminal arbor. The number of branches per unit length was then divided 


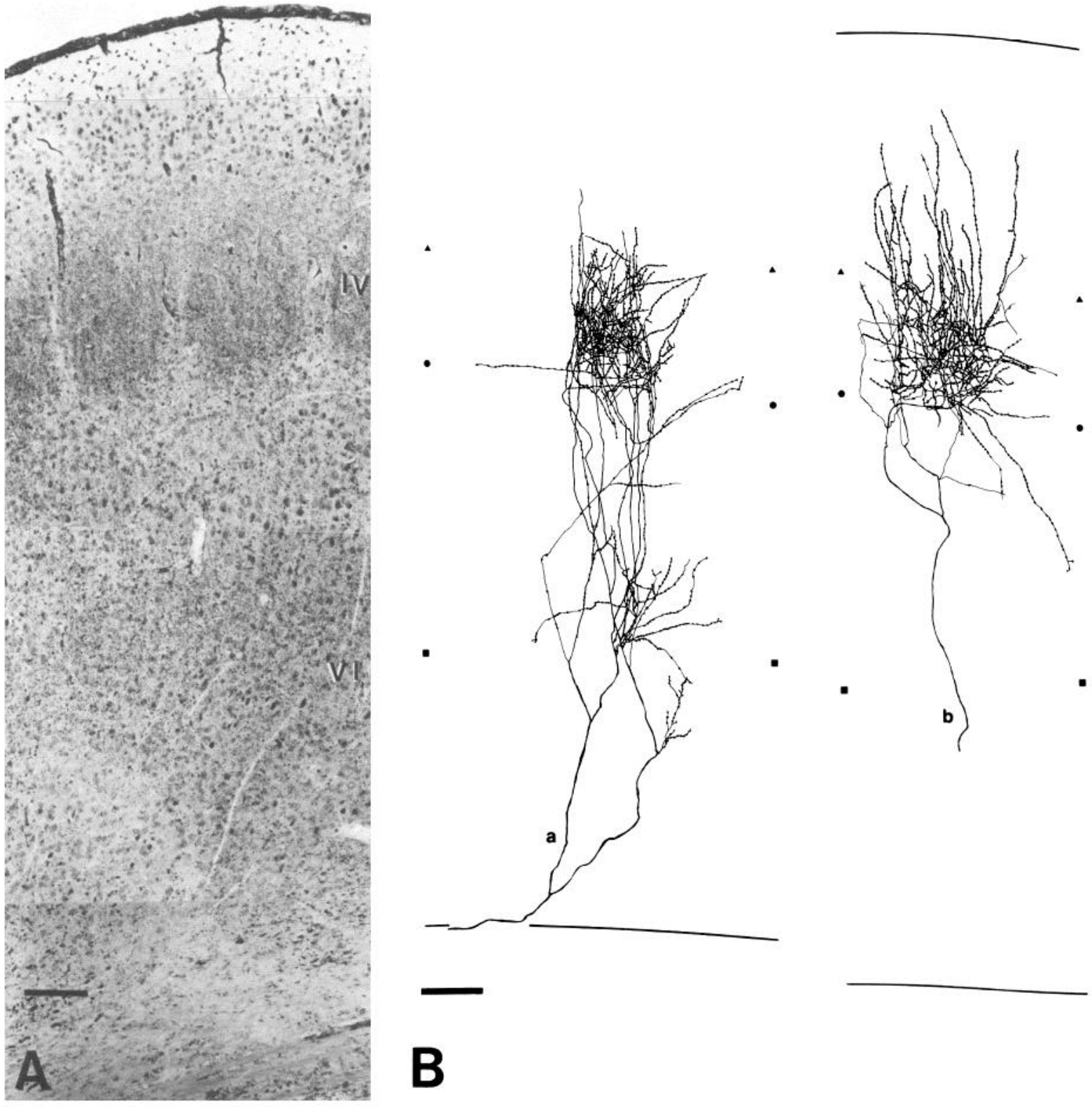

Figure 3. A, The laminar pattern of terminal degeneration in coronal sections of the anterolateral region of the somatosensory cortex resulting from lesions of the ventrobasal nucleus. Discrete patches of dense terminal degeneration occur in layer IV (iv). Additional terminal degeneration can be seen in layer VI (vi). Fink-Heimer. Scale bar, $100 \mu \mathrm{m}$. B, Coronal views of reconstructed terminal arbors from the anterolateral region of the somatosensory cortex. The majority of boutons are located in the dense terminal plexus in layer IV. Triangles indicate the border of layers III and IV. Circles indicate the border of layers IV and V. Squares indicate the border of layers V and VI. Lateral is to the left. Scale bar, $100 \mu \mathrm{m}$.

by the anterior to posterior extent of the plexus. This procedure resulted in an estimate of the number of branches that occupy an average cubic millimeter of the terminal field.

Descriptive statistics. The dimensions of the terminal arbors and cytochrome oxidase clusters from the anterolateral and posteromedial regions were compared using analysis of variance. Following a $\log -\log$ transformation, the relationship between branching density and projection area was analyzed using Pearson's $r$ for correlation. Estimates of the slope and intercept were determined by linear regression.

\section{Results}

Appearance of bulk-labeled axons

Following WGA-HRP injections into the white matter beneath the somatosensory cortex, fibers that have dense terminal arbors in layer IV are labeled (Figs. 1, 2). The parent fibers arising from the white matter are approximately $1-4 \mu \mathrm{m}$ in diameter. They ascend obliquely from the white matter, following a tortuous anterior course through the deeper layers. In some instances, the large-caliber parent fibers branch in layer VI, giving rise to both large- and small-caliber branches. In coronal sections, the larger-caliber branches appear to run parallel to one another to layer IV (Figs. 3B, 5-9). When fine-caliber branches can be followed for an extended distance, they turn toward the pia and ascend to layer IV. Few, if any, collaterals have branches or boutons in layer $\mathrm{Vb}$. In layer $\mathrm{Va}$, numerous radially oriented branches arise from the parent fibers. These branches give off 

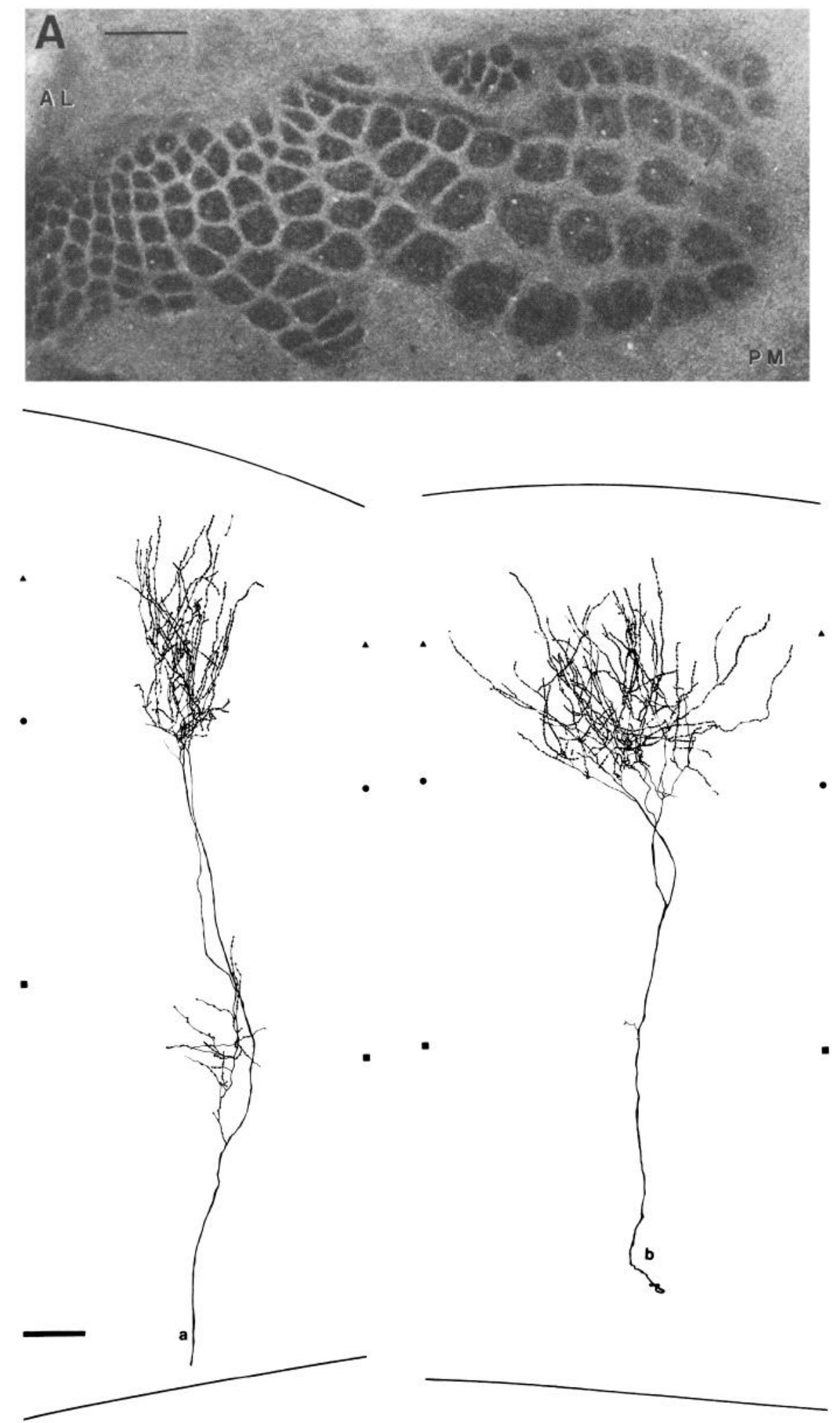

Figure 4. The pattern of succinate dehydrogenase activity in tangential sections of the vibrissae representation of SmI. The anterolateral $(A L)$ segments (left) are smaller than the posteromedial $(P M)$ segments (right). Scale bar, $1 \mathrm{~mm}$. $B, a$, Coronal view of a terminal arbor from the anterolateral region of the somatosensory cortex, with a layer IV plexus that extends $200 \mu \mathrm{m}$ in both mediolateral and anteroposterior dimensions. $b$, Coronal view of a terminal arbor from the posteromedial region with a layer IV plexus extending $530 \mu \mathrm{m}$ in the mediolateral dimension and $300 \mu \mathrm{m}$ in the anteroposterior dimension. Scale bar, $100 \mu \mathrm{m}$. fine collaterals in layer IV, which form a characteristic dense plexus and extend into layer III. The density of collaterals, however, is much lower in layer III, and only a few fine-caliber collaterais reach layer II. Thus, the greatest branching density of these terminal arbors occurs in layer IV. The lateral extension of branches in other layers does not usually exceed the width of the layer IV plexus.

In material reacted with diaminobenzidine, a background staining occurs that parallels the distribution of cytochrome oxidase activity (Figs. 1, 2). Patches of this cytochrome-like 
Table 1. Dimensions of the layer IV plexus of individual terminal arbors

\begin{tabular}{llllll} 
& & $\begin{array}{l}\text { Branch } \\
(\mathrm{b}) \\
\text { density } \\
\left(\mathrm{b} / \mathrm{mm}^{3}\right)\end{array}$ & $\begin{array}{l}\text { Medio- } \\
\text { lateral } \\
\text { extent } \\
(\mathrm{mm})\end{array}$ & $\begin{array}{l}\text { Antero- } \begin{array}{l}\text { Pro- } \\
\text { pos- } \\
\text { terior } \\
\text { extent } \\
(\mathrm{mm})\end{array} \\
\text { Figure } \\
\text { tion } \\
\text { area } \\
\left(\mathrm{mm}^{2}\right)\end{array}$ \\
\hline Anterolateral region & $3 B, a$ & 994 & 0.20 & 0.20 & 0.03 \\
& $3 B, b$ & 415 & 0.25 & 0.30 & 0.06 \\
& $4 B, a$ & 818 & 0.20 & 0.20 & 0.03 \\
& $5 a$ & 534 & 0.15 & 0.18 & 0.02 \\
Posteromedial region & $5 b$ & 436 & 0.30 & 0.24 & 0.06 \\
& $5 c$ & 575 & 0.30 & 0.18 & 0.04 \\
& 8 & 622 & 0.35 & 0.25 & 0.07 \\
& $9 a$ & 433 & 0.30 & 0.20 & 0.05 \\
& $9 b$ & 228 & 0.30 & 0.30 & 0.07 \\
& $6 a$ & 264 & 0.53 & 0.30 & 0.13 \\
Other regions & $6 b$ & 392 & 0.50 & 0.40 & 0.16 \\
& $6 c$ & 204 & 0.45 & 0.30 & 0.11 \\
& $7 a$ & 197 & 0.50 & 0.50 & 0.11 \\
& $7 b$ & 222 & 0.60 & 0.45 & 0.21 \\
& $5 d$ & 934 & 0.10 & 0.23 & 0.02 \\
& $7 c$ & 120 & 0.93 & 0.44 & 0.37 \\
\hline
\end{tabular}

staining are evident in layer IV, and a lighter band was also visible in layer VI. Since the pattern of segments of the vibrissae representation are clearly demonstrated with mitochondrial stains, such as cytochrome oxidase, this background staining is useful in identifying well-filled axons whose terminal arbors are associated with the segments.

In addition to the anterograde labeling of fibers in the neocortex, retrogradely labeled neurons were observed in a number of locations, including the ventral posterior nucleus.

\section{Comparison of the distribution of boutons of bulk-labeled fibers with terminal degeneration following lesions of the ventral posterior nucleus}

A striking feature of the "bulk-labeled" axons was the appearance of boutons as enlargements along fine-calibrated fibers (Fig. 2 ). These boutons vary in size and appear most dense along the smallest-caliber fibers. The overwhelming majority of boutons are on the collaterals of the layer IV plexus (Figs. 3B, 5-9). We have not discerned a differential distribution of boutons within the layer IV plexus of the terminal arbors. Rather, the most complex arbors appear to have a fairly uniform distribution of boutons throughout the layer IV plexus (Figs. 3B, 5-7). A lower density of boutons was found on branches extending into layer III. Boutons also occurred on the sparsely distributed branches in layer II. While there were few fine-caliber branches in layer VI, they generally exhibited numerous boutons along their length within layer VI, while only a few boutons were observed in layer $\mathrm{V}$ (Figs, $3 B, 5-7$ ).

When the ventral posterior nucleus of the thalamus was lesioned and the somatosensory cortex stained by the Fink-Heimer method (Fig. $3 A$ ), dense concentrations of terminal degeneration were apparent in discrete patches in layer IV. This terminal degeneration extends less densely into layer III, and, to a slight extent, into layer II. In addition, some terminal degeneration also occurs in layer VI. Thus, the pattern of terminal degeneration in the somatosensory cortex following lesions of the ventral posterior nucleus precisely parallels the pattern of distribution of boutons found on fibers that have a dense terminal arbor in layer IV.

\section{Distribution of terminal arbors of different sizes within the primary somatosensory cortex}

The pattern of vibrissae-related clusters in the rat somatosensory cortex is clearly revealed by the pattern of succinate dehydrogenase activity (Fig. $4 A$ ). Within this representation, the sinus hairs located anteriorly on the face are represented by small clusters in the anterolateral aspect of SmI. In contrast, the vibrissae located more posteriorly on the face are represented by large clusters located within the posteromedial aspect of SmI. When cytochrome oxidase clusters were measured along an axis equivalent to the coronal plane of section, clusters of the posteromedial vibrissae representation ranged in their mediolateral cxtcnt from approximately 350 to $450 \mu \mathrm{m}$. In the anteroposterior dimension these clusters ranged from 450 to $600 \mu \mathrm{m}$. The clusters of the anterolateral sinus hair representation ranged in their mediolateral extent from approximately 150 to $350 \mu \mathrm{m}$. These fibers ranged from 200 to $400 \mu \mathrm{m}$ in the anteroposterior dimension.

The mediolateral extent of the layer IV plexus of terminal arbors from coronal sections of the anterolateral region of SmI range in size from 150 to $350 \mu \mathrm{m}$ (Table !, Figs. $3 B, 5$ ). The anteroposterior extent of these anterolateral arbors range from 180 to $300 \mu \mathrm{m}$. The mediolateral extent of the layer IV plexus of terminal arbors from the posteromedial region, corresponding to the mystacial vibrissae representation, range from 450 to 600 $\mu \mathrm{m}$ (Table 1, Figs. $4 B, b, 6,7$ ). The anteroposterior extent of these posteromedial arbors range from 300 to $500 \mu \mathrm{m}$. The narrowest arbor reconstructed has a mediolateral extent of 100 $\mu \mathrm{m}$ (Fig. $5 d$ ) and is located laterally, in a region corresponding to the SmII cortex (Carvell and Simons, 1986). The largest terminal arbor reconstructed, which extends $930 \mu \mathrm{m}$ in the mediolateral dimension and $440 \mu \mathrm{m}$ in the anteroposterior dimension (Fig. 7c), is located posteromedial to the vibrissae representation, corresponding to the representation of the trunk. In one of the fibers reconstructed from the anterolateral region, a collateral enters layer II approximately $650 \mu \mathrm{m}$ medially from the layer IV plexus of this terminal arbor (Fig. 8). The boutons associated with this collateral are sparsely distributed throughout the neocortical layers and do not appear to be concentrated in layer IV. When 2 fibers were observed to terminate in the same localized area, their terminal fields appeared to be largely coextensive in the coronal plane (Fig. 9).

The dimensions of both the cytochrome oxidase clusters and the terminal arbors were significantly different in the 2 regions of the somatosensory cortex (Table 2). The size of the cytochrome oxidase clusters parallels the size of the terminal arbors from the corresponding region. Furthermore, when the approximate location and size of the terminal arbors were plotted on a surface view of the somatosensory cortex, the relative size of the arbors correspond to the location of the different-sized vibrissae-related clusters.

\section{Relationship of projection area and branch density}

The dimensions of the layer IV plexus of each of the reconstructed arbors are given in Table 1. The size of the projection area of the terminal arbors in the posteromedial region is significantly larger than the area of those in the anterolateral region 


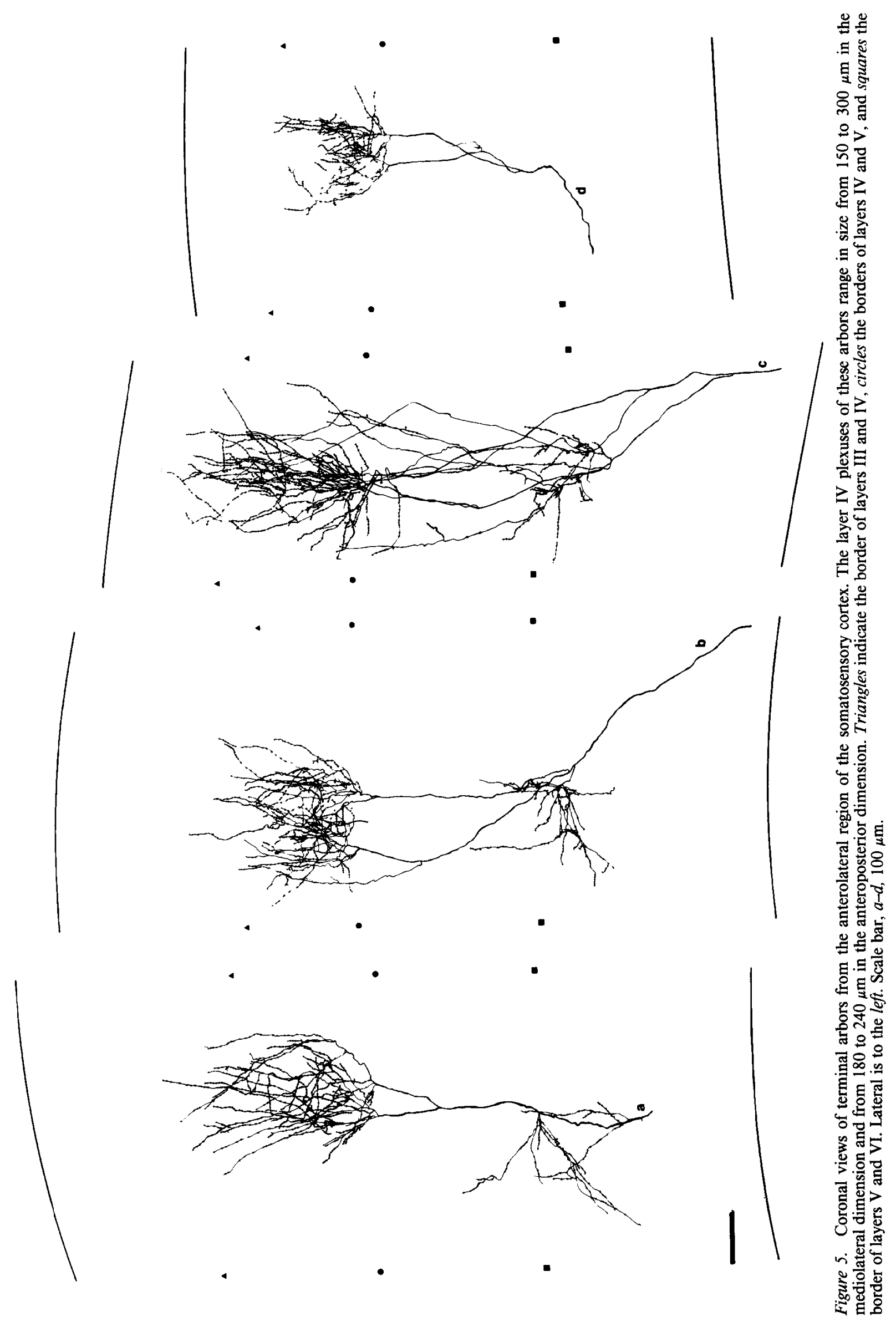




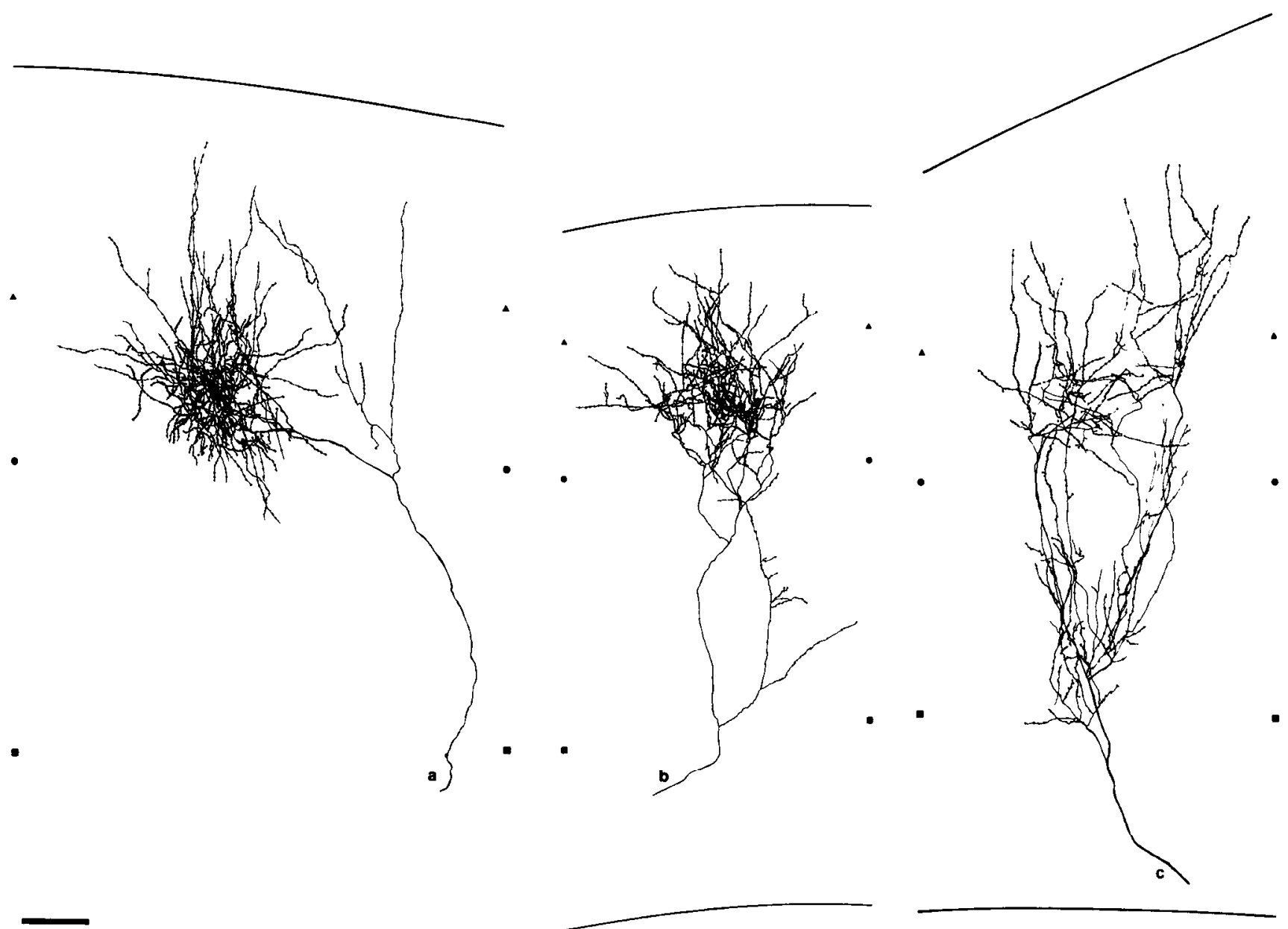

Figure 6. Coronal views of terminal arbors from the posteromedial region of the somatosensory cortex. These arbors are located in the region corresponding to the representation of the mystacial vibrissae. The layer IV plexuses of these terminal arbors range in size from 450 to $500 \mu \mathrm{m}$ in the mediolateral dimension and from 300 to $400 \mu \mathrm{m}$ in the anteroposterior dimension. Scale bar, $a-c, 100 \mu \mathrm{m}$.

Table 2. Dimensions of layer IV plexuses of terminal arbors and cytochrome oxidase clusters in the anterolateral and posteromedial regions of the somatosensory neocortex

\begin{tabular}{|c|c|c|c|}
\hline & $\begin{array}{l}\text { Antero- } \\
\text { lateral } \\
\text { region } \\
(n=9) \\
\end{array}$ & $\begin{array}{l}\text { Postero- } \\
\text { medial } \\
\text { region } \\
(n=6) \\
\end{array}$ & $p^{a}$ \\
\hline \multicolumn{4}{|c|}{ Layer IV terminal plexuses of bulk-filled fibers } \\
\hline Branching density $\left(\mathrm{b} / \mathrm{mm}^{3}\right)$ & $562 \pm 72$ & $248 \pm 28$ & $<0.01$ \\
\hline Anteroposterior extent (mm) & $0.26 \pm 0.02$ & $0.51 \pm 0.02$ & $<0.001$ \\
\hline Mediolateral extent (mm) & $0.23 \pm 0.01$ & $0.38 \pm 0.03$ & $<0.0001$ \\
\hline Projection area $\left(\mathrm{mm}^{2}\right)$ & $0.05 \pm 0.01$ & $0.15 \pm 0.02$ & $<0.0001$ \\
\hline \multicolumn{4}{|c|}{ Cytochrome oxidase-stained clusters } \\
\hline Anteroposterior extent (mm) & $0.21 \pm 0.01$ & $0.44 \pm 0.01$ & $<0.0001$ \\
\hline Mediolateral extent (mm) & $0.28 \pm 0.02$ & $0.56 \pm 0.03$ & $<0.0001$ \\
\hline Projection area $\left(\mathrm{mm}^{2}\right)$ & $0.05 \pm 0.01$ & $0.19 \pm 0.01$ & $<0.0001$ \\
\hline
\end{tabular}

${ }^{a}$ The probability that differences between anterolateral and posteromedial values occurred by chance (ANOVA). 


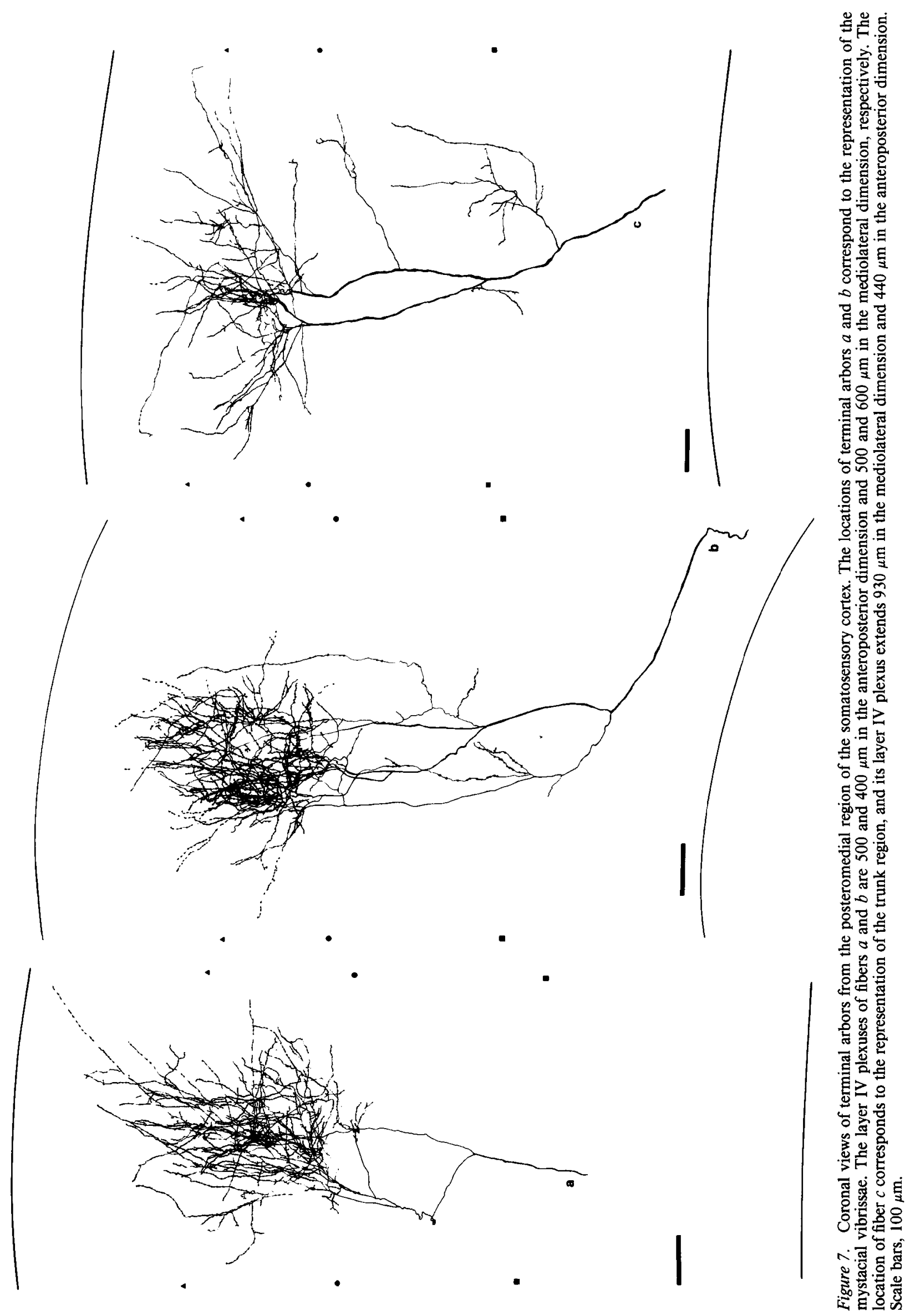




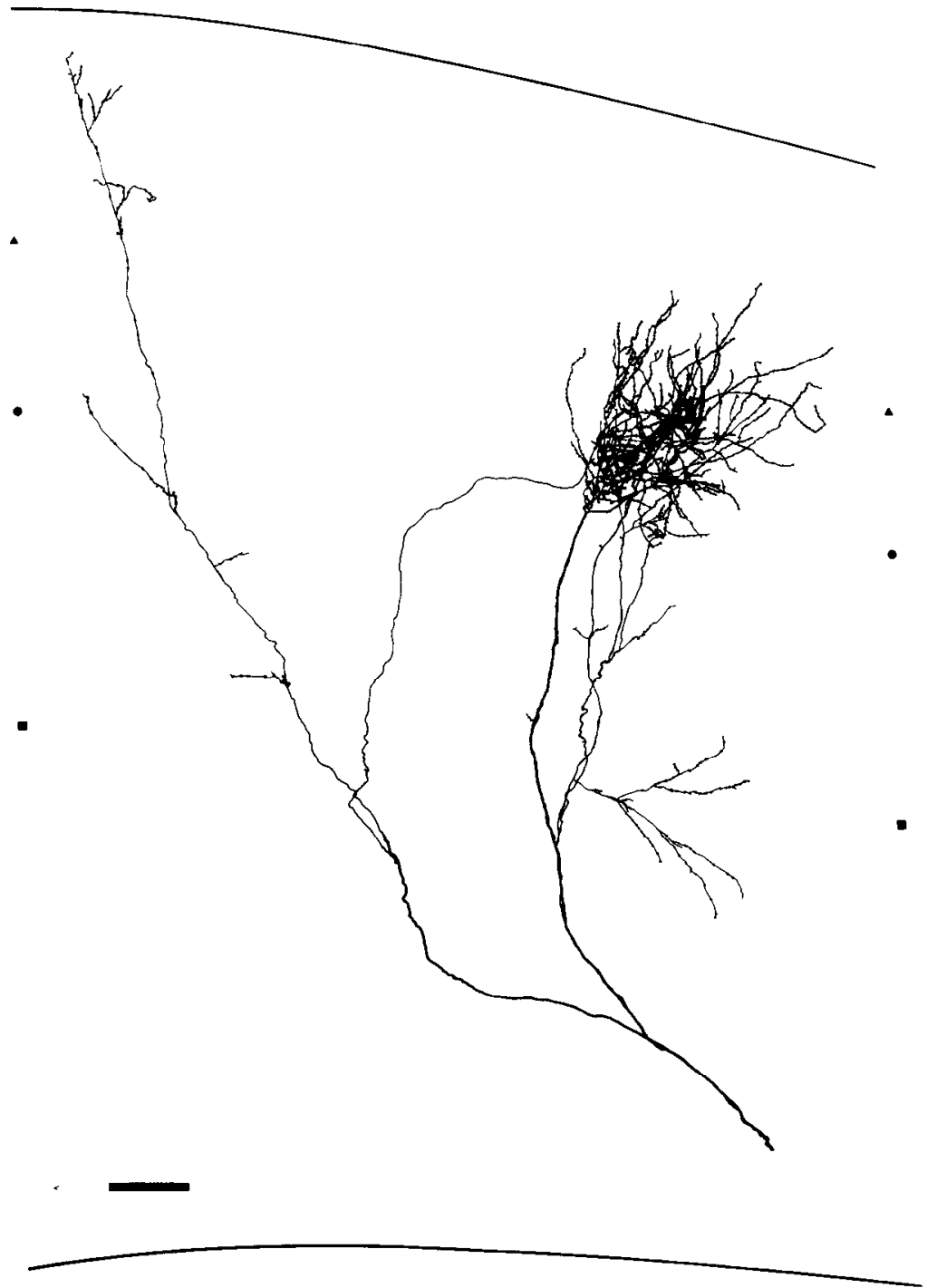

Figure 8 . Coronal view of a terminal arbor of the anterolateral region of the somatosensory cortex that exhibits a branch entering layer II approximately $650 \mu \mathrm{m}$ medially from its dense layer IV plexus. The layer IV plexus extends $350 \mu \mathrm{m}$ in the mediolateral dimension and $250 \mu \mathrm{m}$ in the anteroposterior dimension. Scale bar, $100 \mu \mathrm{m}$.

(Table 2). These differences correspond closely to the differences in the size of cytochrome oxidase clusters from the 2 regions (Table 2). Conversely, the branch density is significantly greater in the terminal arbors of the anterolateral region than in the posteromedial region. The relationship between projection area and branch density for all terminal arbors examined is described by the hyperbolic function,

$$
\text { Branch density }=67.6 *(\text { projection area })^{-0.65} \text {. }
$$

A $\log -\log$ transformation yields a linear model $(p<0.001)$ :

$$
\log (\text { branch density })=-0.65 \cdot \log (\text { projection area })+1.83 \text {. }
$$

The higher-order quadratic and cubic models are not significant, $(p>0.8)$. The log of the branch density is negatively correlated with the $\log$ of the projection area $(r=-0.90, p<0.0001$; Fig. 10).

\section{Correspondence between neocortical clusters and afferent terminal arbors}

The direct correspondence between the terminal arbors of individual afferent fibers and vibrissae-related neocortical clusters was examined by reconstructing terminal arbors from tangential sections stained simultaneously for cytochrome oxidase activity. The large number of fine-caliber fibers that had to be aligned between adjacent sections made reconstruction across tangential sections particularly difficult. One partial (Fig. $11 B, b$ ) and one more completely reconstructed terminal arbor (Fig. $11 B, a$ ) were obtained. These were located in clusters corresponding to the representation of the mystacial vibrissae (Fig. 11A). The tortuous anterior course of the parent fiber as it leaves the white matter (Fig. $11 B, b$, arrow) and runs through the deeper layers is obvious in tangentially reconstructed sections. The majority of the boutons of each of these axonal arbors remain within a single segment. In one case (Fig. $11 A, a$ ), a few branches exhibiting boutons reach adjacent clusters within the row.

The appearance of terminal arbors in tangential sections is consistent with the appearance of the projection areas of reconstructed fibers plotted on a surface view of the somatosensory neocortex (Fig. 12). Two features of the terminal arbors are apparent when the data are viewed this way. First, there are consistent differences in the size of projection areas in the anterolateral and posteromedial regions of the somatosensory neo- 


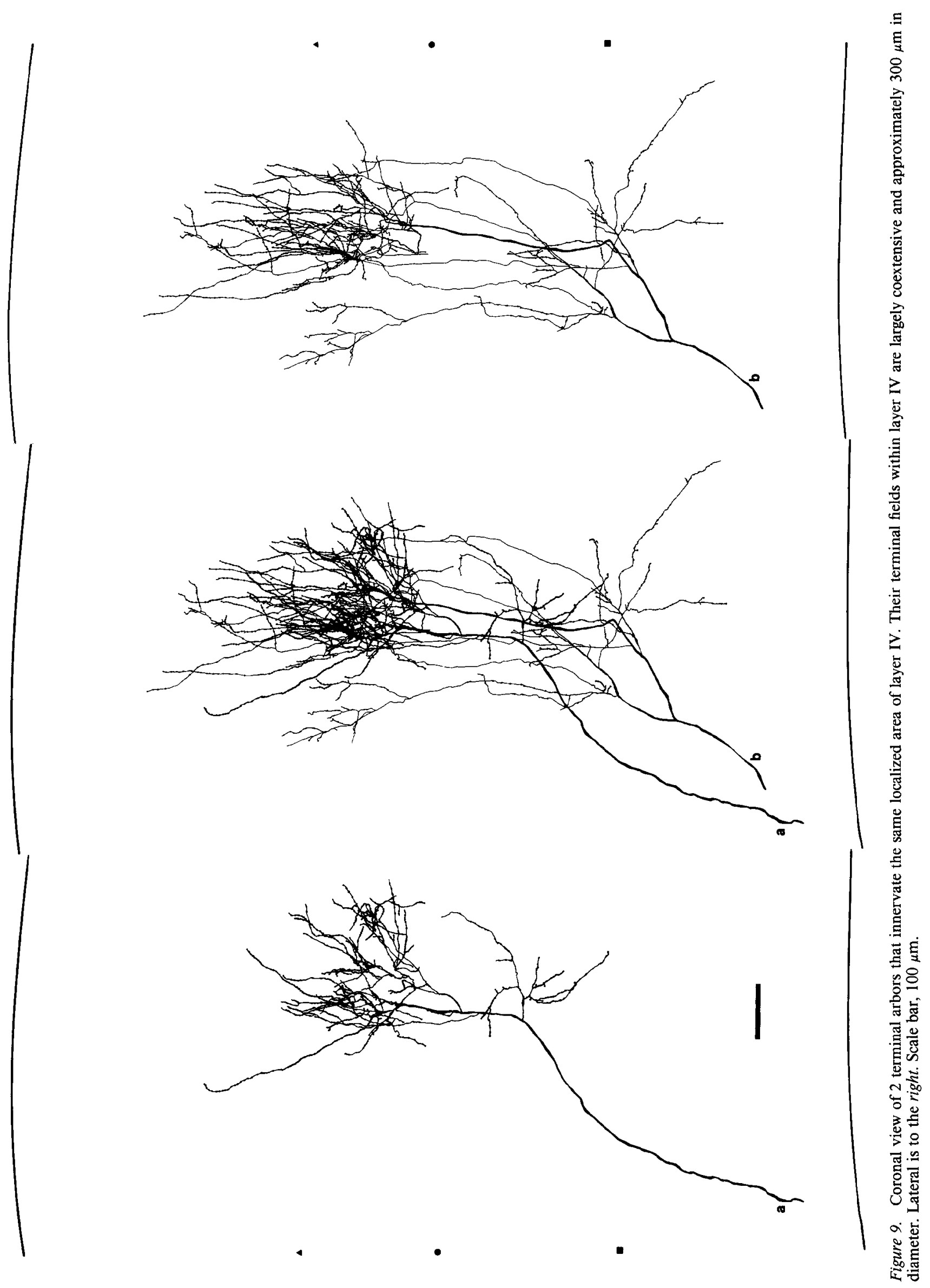




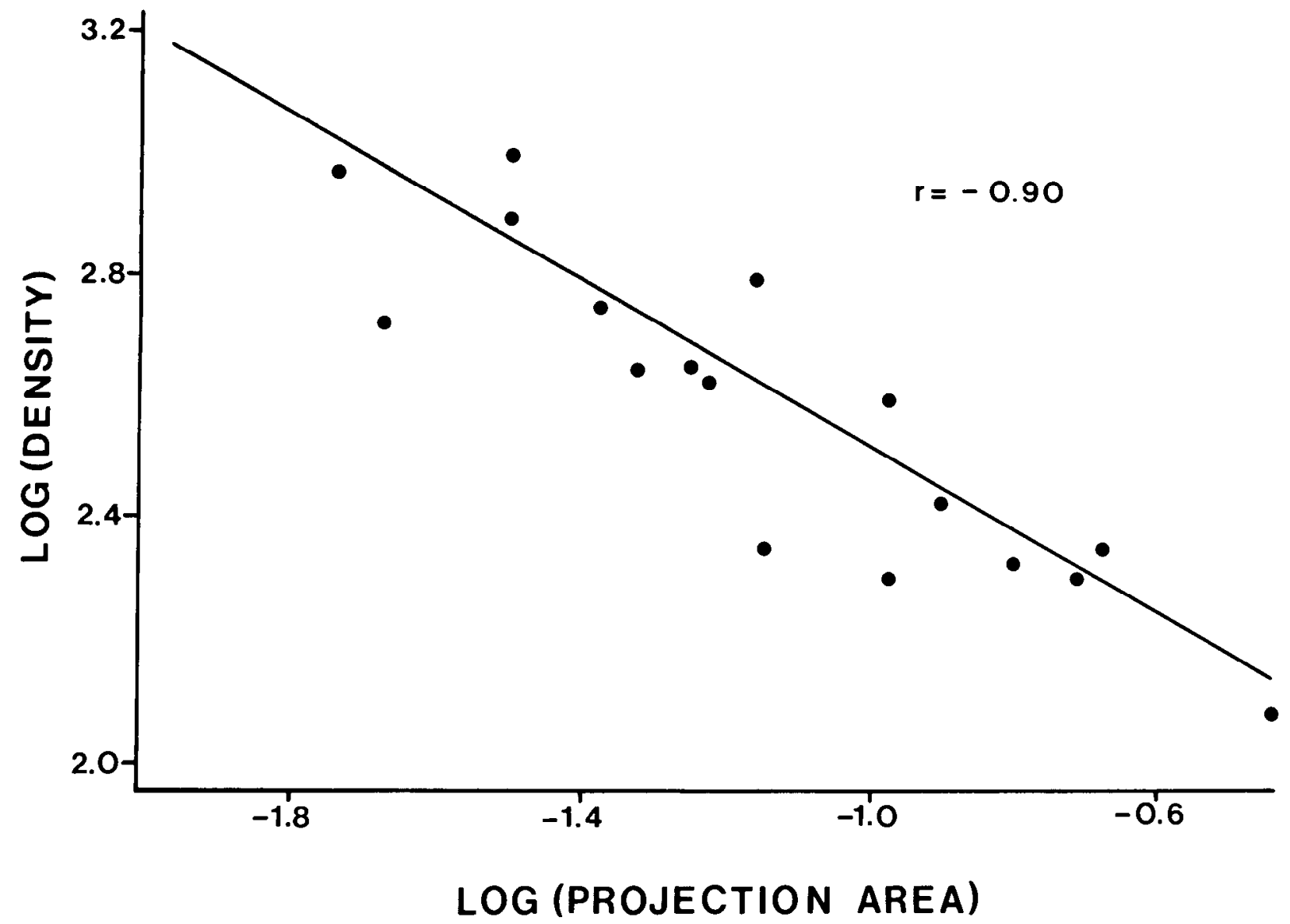

Figure 10. A $\log -\log$ plot of density of branches (density) in the layer IV plexus of reconstructed terminal arbors versus the area of projection onto layer IV (projection area). All reconstructed terminal arbors are included in this analysis (see Table 1). Pearson's $r=-0.90, p<0.0001$. The probability that the linear relationship occurred hy chance is less than 0.0001 . The intercept equals $1.83+0.10$ and the slope equals $-0.65+0.08$ $(p<0.0001)$.

cortex. Second, there is a close correspondence between the size of the projection ared of a layer IV plexus and that of cylochrome oxidase clusters in the corresponding region.

\section{Discussion}

In this report we have described the morphology of axons "bulklabeled" by injection of WGA-HRP into the white matter beneath the somatosensory cortex of the rat. We conclude that these axons, which are characterized by a dense layer IV terminal plexus, originate in the ventral posterior nucleus and correspond to the "specific" thalamocortical afferents first described by Lorente de No $(1922,1949)$. This conclusion is based on the following evidence. First, the laminar distribution of boutons on these terminal arbors corresponds to the laminar distribution of terminal degeneration following lesions of the ventral posterior nucleus, the pattern of anterogradely transported tritiated amino acids from the ventral posterior nucleus (Herkenham, 1980), and the pattern of anterogradely transported Phaseolus vulgaris lectin (Keller et al., 1985). In addition, we have observed retrogradely labeled neurons in the ventral posterior nucleus following the white matter injections that anterogradely labeled these afferent arbors. Second, the regional distribution of arbors of different size corresponds to the regional variation in cluster size, as demonstrated with succinate dehydrogenase histochemistry.
Our observations indicate that in the rat somatosensory cortex, the plexus of an individual specific thalamocortical afferent occupies the full vertical width of layer IV and extends into layer III and, to a much lesser extent, into layer II. This result is, to a large extent, in accord with a previous report on terminal arbors in the somatosensory cortex of the cat (Landry and Deschenes, 1981). However, it contrasts with reports of the segregated distribution of terminals within subzones of layer IV in the visual cortex of several different species. In both the monkey (Blasdel and Lund, 1983) and the tree shrew (Conley et al., 1984), given laminac of the lateral geniculate project to specific subzones of layers IV and III. In the lateral geniculate of the cat, functionally distinct populations of neurons are intermixed in a given layer, but appear to project differentially to specific subzones of layers IV and III (Humphrey et al., 1985a). Thus, the sublaminar specificity of geniculocortical axons appears to be related to the processing of visual information. The apparent lack of such sublaminar specificity in the somatosensory system suggests that there may be differences in the processing requirements of different sensory systems.

In the somatosensory (Landry and Deschenes, 1981) and visual cortices (Ferster and Levay, 1978; Gilbert and Wiesel, 1983; Humphrey et al., 1985a, b) of the cat, as well as the visual cortex of the monkey (Blasdel and Lund, 1983), many terminal arbors of thalamic axons appear to have multiple patches of dense 


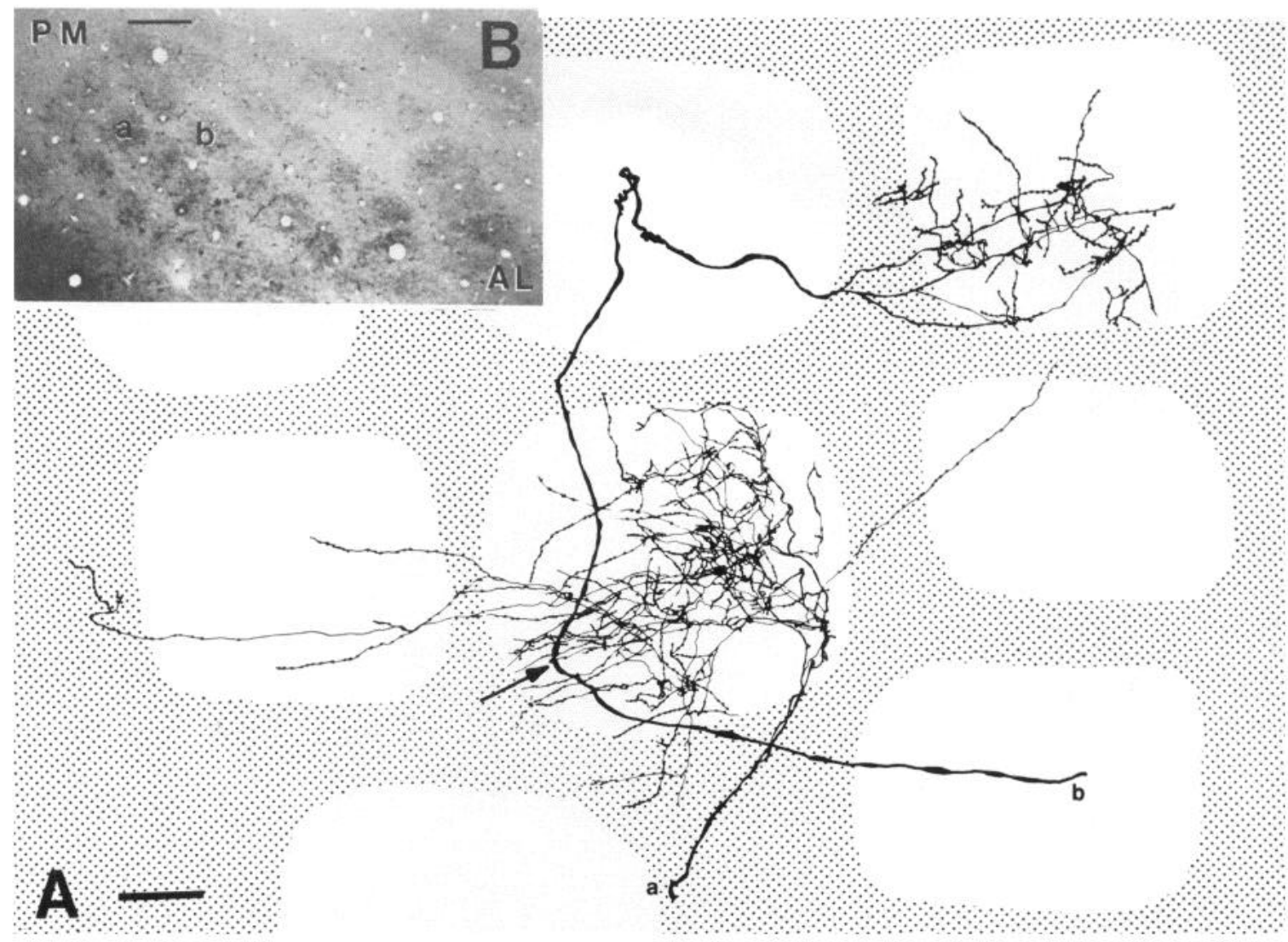

Figure 11. A, Fibers reconstructed from tangential sections of the posteromedial portion of the face region stained simultaneously for HRP and cytochrome oxidase activity. Stippling represents the area between clusters of cytochrome oxidase activity. The arrow indicates where fiber $a$ emerges from the white matter. Scale bar, $100 \mu \mathrm{m}$. B. Low-power photomicrograph of one of the tangential sections from which fibers $a$ and $b$ were reconstructed. The clusters of cytochrome oxidase activity in which the reconstructed fibers of Figure $A$ have their dense plexuses are indicated by $a$ and $b$, respectively. $P M$ and $A L$, posteromedial and anterolateral aspects of the mystacial vibrissae representation, respectively. Scale bar, $500 \mu \mathrm{m}$.

terminal arbors separated by zones relatively devoid of boutons. In the visual system, these patches can be clearly related to functionally defined ocular dominance columns (Blasdel and Lund, 1983; Gilbert and Wiesel, 1983). The significance of such multiple patches in the somatosensory cortex of the cat is obscure. In the present study, we found no evidence of such multiple patches. While we cannot rule out the possibility that individual axons branch along their course within the internal capsule, several lines of evidence indicate that the failure to label multiple patches is not due to technical limitations. One, the labeled fibers represented were considered to be well filled since collaterals generally exhibited terminal boutons. Two, the laminar pattern of boutons appears complete when compared with the pattern of anterograde degeneration that follows lesions of the ventral posterior nucleus and the pattern of anterogradely transported amino acids from the ventral posterior nucleus (Herkenham, 1980). In particular, fine collaterals bearing terminal boutons could be detected in layer VI. Three, patches appear most distinct when arbors are viewed tangential to the cortical surface (Blasdel and Lund, 1983; Gilbert and Wiesel, 1983). The well-filled arbor reconstructed in the tangential plane did not exhibit multiple patches of boutons. Four, when the appearance of anterograde label of WGA-HRP from the ventrobasal complex to $\mathrm{SmI}$ is compared in the vibrissae and trunk regions, we find that the label in the vibrissae region exhibited its characteristic clustered organization, while the label in the trunk region appeared continuous. The size of the terminal arbors in these 2 different regions varied in a corresponding fashion. We attribute the absence of such multiple patches on the axons we observed in rat SmI to be a consequence of the discrete character of the rodent trigeminal system as compared to the more continuous character of the visual fields and some portions of the body surface. We therefore interpret our findings as indicating that the individual terminal arbors from the ventral posterior nucleus terminate in a discrete pattern that is reflected in the vibrissae-related clusters in SmI. Thus, the one vibrissaone cluster relationship is an expression of the size and distribution of individual arbors of thalamocortical axons.

It should also be noted that not all geniculate axons terminate in multiple patches. In the cat visual cortex, the majority of X-cell terminations form a single patch (Humphrey et al., 1985a, b), while in monkey visual cortex, a type of axon projecting to layer $4 \mathrm{C}$ beta has a highly circumscribed terminal arbor. Indeed, Blasdel and Lund (1983) suggest that the size of the discrete arbors terminating in layer $4 \mathrm{C}$ beta is the most likely candidate underlying the high degree of retinotopic specificity characteristic of the macaque monkey striate cortex.

With regard to the tangential dimension of the cortex, we have observed that the projection area of a terminal arbor varies in size depending on the portion of the cortical map to which 

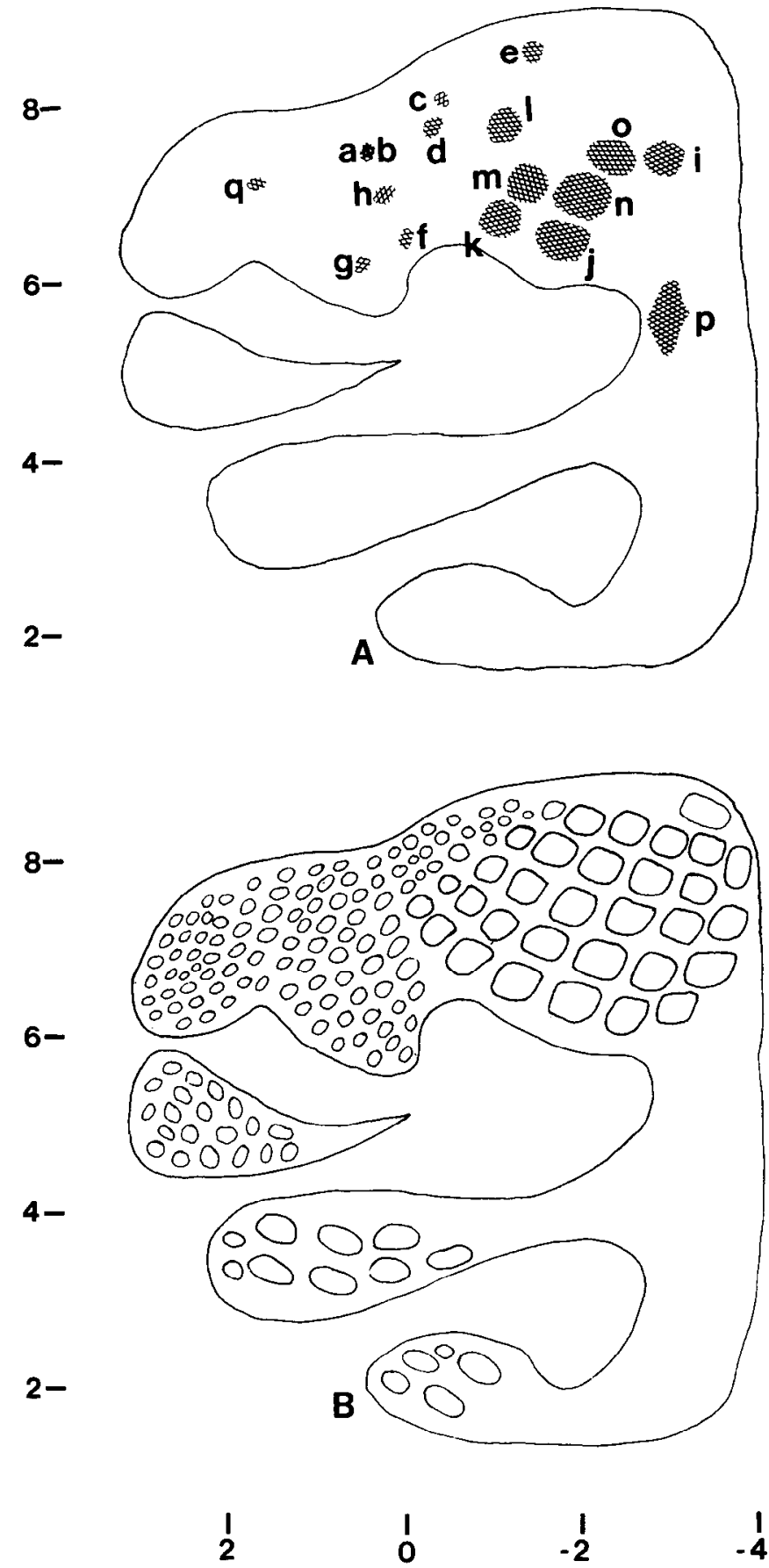

Figure 12. A, Diagrammatic representation of the approximate size and location of the layer IV plexus of the reconstructed terminal arbors represented in the previous figures. The outlines represent the approximate orientations of the different body parts within the somatotopic map. Fields within the anterolateral portion of the face region: $a$ and $b$, coextensive terminal arbors represented in Higure $9 ; c$, arbor represented in Figure $3 B a ; d, 3 B b ; e$, arbor represented in $4 B a ; f, 5 a, g, 5 b$; $h .5 c ; q .8$. Fields within the posteromedial portion of the face region: $i$ corresponds to layer IV plexus of the arbor represented in Figure $11 \mathrm{~A}$, $a ; j, 4 B b ; k, 6 a ; l, 6 b ; m, 6 c ; n, 7 a ; 0,7 b$. Field $p$ represents the layer IV plexus of the arbor located in the region corresponding to the trunk representation, Figure $7 c . B$, Diagrammatic representation of succinate dehydrogenase activity in flattened sections of somatosensory cortex, indicating the pattern of clustering of thalamocortical projcctions for comparison with $A$. Horizontal scale, millimeters from bregma; vertical scale, millimeters lateral from the midline.

it is related. Furthermore, arbors that have a larger projection area have a lower density of branches. This observation is particularly significant in light of previous findings that the number of neurons in a neocortical "barrel," and consequently its size, is proportional to the number of myelinated axons innervating the corresponding vibrissa (Lee and Woolsey, 1975; Welker and Van der Loos, 1986). Taken together, these observations suggest 2 organizing principles governing thalamocortical projections. First, the consistent relationship between projection area and branching density for all terminal arbors examined is consistent with the homogeneity of thalamocortical projection neurons in the ventrobasal nucleus of the rat (Harris, 1986). The relationship between these 2 aspects of terminal arbor morphology may therefore be, to some degree, intrinsically regulated by thalamocortical projection neurons. Second, peripheral influences play a significant role in determining both the characteristic size and density of the layer IV plexus. The quantitative relationship between ncocortical cluster sizc and peripheral innervation density suggests at least 2 possible ways in which the periphery may influence thalamocortical projection neurons. One possibility is that smaller vibrissae-related clusters receive axons from fewer thalamic projection neurons and, as a consequence, each thalamocortical neuron contributes more branches to the cluster. This is unlikely, however, since a uniform reduction in the number of primary afferents does not significantly alter the size of whisker-related clustering (Sikich et al., 1986). Another possibility is that the periphery may influence the rate of branching as an axon grows into layer IV. A greater degree of branching would result in a smaller projection area, while a lesser degree of branching would result in a larger one.

In summary, terminal arbors of specific thalamocortical axons vary in size and branching density according to their regional distribution. These characteristics of the thalamocortical terminal arbors parallel peripheral innervation density. Thus, terminal arbor morphology may underlie the peripherally dependent characteristics of the neocortical somatotopic map.

\section{References}

Amaral, D. G., and J. L. Price (1983) An air pressure system for the injection of tracer substances into the brain. J. Neurosci. Methods 9 : $35-43$.

Bates, C. A., and H. P. Killackey (1985) The organization of the neonatal rat's brainstem trigeminal complex and its role in the formation of central trigeminal patterns. J. Comp. Neurol. 240:265287.

Bates, C. A., R. S. Erzurumlu, and H. P. Killackey (1982) Central correlates of peripheral pattern alterations in the trigeminal system of the rat. III. Neurons of the principal sensory nucleus. Dev. Brain Res. 5: 108-113.

Belford, G. R., and H. P. Killackey (1979a) Vibrissae representation in subcortical trigeminal centers of the neonatal rat. J. Comp. Neurol. 183: 305-322.

Belford, G. K., and H. P. Killackey (1979b) The development of vibrissae representation in subcortical trigeminal centers of the neonatal rat. J. Comp. Neurol. 183: 63-74.

Belford, G. R., and H. P. Killackey (1980) The sensitive period in the development of the trigeminal system of the neonatal rat. J. Comp. Neurol. 193: 335-350.

Blasdel, G. G., and J. S. Lund (1983) Termination of afferent axons in macaque striate cortex. J. Neurosci. 3: 1389-1413.

Carvell, G. G., and D. J. Simons (1986) Somatotopic organization of the second somatosensory area (SII) in the cerebral cortex of the mouse. Somatosensory Res. 3: 213-237.

Chapin, J. K., and C.-S. Lin (1984) Mapping the body representation in the SI cortex of anesthetized and awake rats. J. Comp. Neurol. 229: 199-213.

Conley, M., D. Fitzpatrick, and I. T. Diamond (1984) The laminar 
organization of the lateral geniculate body and the striate cortex in the tree shrew (Tupaia glis). J. Neurosci. 4: 171-197.

Dawson, D. R., and H. P. Killackey (1987) The organization and mutability of the forepaw and hindpaw representations in the somatosensory cortex of the neonatal rat. J. Comp. Neurol. (in press).

Erzurumlu, R. S., and H. P. Killackey (1983) Development of order in the rat trigeminal system. J. Comp. Neurol. 213: 365-380.

Ferster, D., and S. Levay (1978) The axonal arborizations of lateral geniculate neurons in the striate cortex of the cat. J. Comp. Neurol. 182: 923-944.

Gilbert, C. D., and T. N. Wiesel (1983) Clustered intrinsic connections in cat visual cortex. J. Neurosci. 3: 1116-1133.

Harris, R. M. (1986) Morphology of physiologically identified thalamocortical relay neurons in the rat ventrobasal thalamus. J. Comp. Neurol. 251: 491-505.

Herkenham, M. (1980) Laminar organization of thalamic projections to the rat neocortex. Science 207: 532-535.

Humphrey, A. L., M. Sur, D. J. Ulrich, and S. M. Sherman (1985a) Projection patterns of individual $\mathrm{X}$ - and $\mathrm{Y}$-cell axons from the lateral geniculate nucleus to cortical area 17 in the cat. J. Comp. Neurol. 233: $159-189$

Humphrey, A. L., M. Sur, D. J. Ulrich, and S. M. Sherman (1985b) Termination patterns of individual $\mathrm{X}$ - and $\mathrm{Y}$-cell axons in the visual cortex of the cat: Projections to area 18 , to the $17 / 18$ border region, and to areas 17 and 18. J. Comp. Neurol. 233: 190-212.

Itoh, K., A. Konishi, S. Normura, N. Mizuno, Y. Nakamura, and T. Sugimoto (1979) Application of coupled oxidation reaction to electron microscopic demonstration of horseradish peroxidase: Cobaltglucose oxidase method. Brain Res. 175: 341-346.

Keller, A., E. L. White, and P. B. Cipolloni (1985) The identification of thalamocortical axon terminals in barrels of mouse SmI cortex using immunohistochemistry of anterogradely transported lectin (Phaseolus vulgaris-leucoagglutinin). Brain Res. 343: 159-165.

Killackey, H. P. (1973) Anatomical evidence for cortical subdivisions based on vertically discrete thalamic projections from the ventral posterior nucleus to cortical barrels in the rat. Brain Res. 51: 326331.

Killackey, H. P. (1980) Pattern formation in the trigeminal system of the rat. Trends Neurosci. 3: 303-305.

Killackey, H. P.,and G. R. Belford (1979) The formation of afferent patterns in the somatosensory cortex of the neonatal rat. J. Comp. Neurol. 183: 285-304.

Killackey, H. P., and G. R. Belford (1980) Central correlates of peripheral pattern alterations in the trigeminal system of the rat. Brain Res. 183: 205-210.

Killackey, H. P., and S. Leshlin (1975) The organization of specific thalamocortical projections to the posteromedial barrel subfield of the rat somatic sensory cortex. Brain Res. 86: 469-472.

Killackey, H. P., G. R. Belford, R. Ryugo, and D. K. Ryugo (1976) Anomalous organization of thalamocortical projections consequent to vibrissae removal in the newborn rat and mouse. Brain Res. 194: 309-315.
Land, P. W., and D. J. Simons (1985) Cytochrome oxidase staining in the rat SmI barrel cortex. J. Comp. Neurol. 238: 225-235.

Landry, P., and M. Deschenes (1981) Intracortical arborizations and receptive fields of identified ventrobasal thalamocortical afferents to the primary somatic sensory cortex in the cat. J. Comp. Neurol. 199: 345-371.

Lee, K. L., and T. A. Woolsey (1975) A proportional relationship between peripheral innervation density and cortical neuron number in the somatosensory system of the mouse. Brain Res. 99: 349-353.

Lorente de No, R. (1922) La corteza cerebral del raton. Trab. Lab. Invest. Biol. Univ. Madrid 20: 1-38.

Lorente de No, R. (1949) Cerebral cortex: Architectonics, intracortical connections. In Physiology of the Nervous System, 3rd Ed., J. Fulton, ed., pp. 274-301, Oxford U. P., Oxford, U.K.

Mesulam, M.-M. (1982) Tracing Neutral Connections with Horseradish Peroxidase, Wiley, New York.

Rosene, D. L., and M.-M. Mesulam (1978) Fixation variables in horseradish peroxidase neurohistochemistry. $\mathrm{I}$. The effects of fixation time and perfusion procedures upon enzyme activity. J. Histochem. Cytochem. 26: 28-39.

Sikich, L., T. A. Woolsey, and E. M. Johnson, Jr. (1986) Effect of a uniform partial denervation of the periphery on the peripheral and central vibrissal system in guinea pigs. J. Neurosci. 6: 1227-1240.

Simons, D. J. (1978) Response properties of vibrissae units in rat SI somatosensory neocortex. J. Neurophysiol. 41: 798-820.

Simons, D. J., and T. A. Woolsey (1979) Functional organization in mouse barrel cortex. Brain Res. 165: 327-332.

Van der Loos, H., and T. A. Woolsey (1973) Somatosensory cortex: Structural alterations following early injury to sense organs. Science 179: 395-398.

Welker, C. (1971) Microelectrode delineation of fine grain somatotopic organization of SmI cerebral neocortex in albino rat. Brain Res. 26: 259-275.

Welker, C. (1976) Receptive fields of barrels in the somatosensory neocortex of the rat. J. Comp. Neurol. 166: 173-190.

Welker, C., and T. A. Woolsey (1974) Structure of layer IV in the somatosensory neocortex in the rat: Description and comparison with the mouse. J. Comp. Neurol. 158: 473-454.

Welker, E., and H. Van der Loos (1986) Quantitative correlation between barrel-field size and the sensory innervation of the whiskerpad: A comparative study in size strains of mice bred for different patterns of mystacial vibrissae. J. Neurosci. 6: 3355-3373.

Wong-Riley, M. (1979) Changes in the visual system of monocularly sutured or enucleated cats demonstrablc with cytochrome oxidase histochemistry. Brain Res. 171: 11-28.

Woolsey, T. A., and H. Van der Loos (1970) The structural organization of layer IV in the somatosensory region (SI) of the mouse cerebral cortex: The description of a cortical field composed of discrete cytoarchitectonic units. Brain Res. 17: 205-242. 